\title{
Probing Transverse Momentum Dependent Parton Distributions in Charmonium and Bottomonium Production
}

\author{
Asmita Mukherjee and Sangem Rajesh \\ Department of Physics, Indian Institute of Technology Bombay, Mumbai-400076, India.
}

(Dated: November 5, 2018)

\begin{abstract}
We propose the study of unpolarized transverse momentum dependent gluon parton distributions as well as the effect of linearly polarized gluons on transverse momentum and rapidity distributions of $J / \psi$ and $\Upsilon$ production within the framework of transverse momentum dependent factorization employing color evaporation model (CEM) in unpolarized proton-proton collision. We estimate the transverse momentum and rapidity distributions of $J / \psi$ and $\Upsilon$ at LHCb, RHIC and AFTER energies using TMD evolution formalism.
\end{abstract}




\section{INTRODUCTION}

In recent years, transverse momentum dependent parton distributions (TMDs) and fragmentation functions (TMFs) have gained a lot of interest both theoretically and experimentally. These are objects that play an essential role for example in single spin asymmetries, where one of the colliding beams/target is polarized. In order to gain information on TMDs, one needs a process like semi-inclusive deep inelastic scattering (SIDIS) or Drell-Yan (DY), where one observes the transverse momentum of a produced particle. There are interesting theoretical issues associated with the TMDs like universality and factorization [1], and a lot of theoretical work have been done in the past few years to shed light on these issues. However, more experimental data are required to fully understand them. There are interesting and useful data from HERMES, COMPASS, JLab, as well as from Tevatron, LHC, Belle and BaBar collaborations. The ultimate goal is to obtain a global fit for the TMDs using all data. However, the problem lies in the fact that the data from SIDIS are at a low energy compared to the data for DY process [2]. It has been shown that in the range $\Lambda_{Q C D}<<k_{\perp}<<Q$ where $k_{\perp}$ is the transverse momentum and $Q$ is the large momentum scale of the process, radiative gluon emission play an essential role and these need to be resummed, giving rise to evolution of TMDs [3], whereas for low $k_{\perp}$, non-perturbative physics dominates. To get a complete picture, one would need to use a lot of data from different experiments in different kinematics. Here it is important to first investigate the unpolarized TMDs. Not only this is important to understand the behaviour of TMDs in general over a large range of momentum scales, but also it is important for spindependent studies as these lie in the denominator of spin asymmetries. Information on quark and antiquark TMDs can be obtained for example from SIDIS, however, gluon TMDs can be best studied in $p p$ and $p \bar{p}$ collisions.

It has been pointed out [4] that gluons can be linearly polarized even inside an unpolarized hadron provided that gluons should have non-zero transverse momentum with respect to the parent hadron. The gluon-gluon correlation function contains the information regarding linearly polarized gluons. Formally, gluon correlator of the unpolarized spin- $\frac{1}{2}$ hadron is parameterized in terms of leading twist transverse momentum dependent (TMD) distribution functions [5] i.e., $f_{1}^{g}\left(x, \mathbf{k}_{\perp}^{2}\right)$ and $h_{1}^{\perp g}\left(x, \mathbf{k}_{\perp}^{2}\right)$. Here, $f_{1}^{g}$ represents the likelihood of finding an unpolarized gluon 
with longitudinal momentum fraction $x$ and transverse momentum $k_{\perp}$ inside an unpolarized hadron and $h_{1}^{\perp g}$, Boer-Mulders function, describes the distribution of linearly polarized gluons within the unpolarized hadron (time-reversal even, or T-even). In general, quarks can also be transversely polarized within the unpolarized hadron. Quark distribution function $h_{1}^{\perp q}$ is timereversal odd (T-odd) function. Experimentally, large $\cos 2 \phi$ azimuthal asymmetry was observed in DY process [6, 7]. It was also noticed in SIDIS by EMC [8] and ZEUS [9] experiments. As suggested in [10], the observed $\cos 2 \phi$ azimuthal asymmetry can be explained by Boer-Mulders effect. Quark Boer-Mulders function $\left(h_{1}^{\perp q}\right)$ has been explored in SIDIS process [11] assuming a relation with Sivers function $f_{1 T}^{\perp q}[12,13]$. It was noticed in [11] that Cahn effect [14, 15] also leads to the $\cos 2 \phi$ asymmetry with a comparable contribution as that of Boer-Mulders effect. Moreover, antiquark Boer-Mulders function was estimated in DY process [16] by measuring azimuthal asymmetry. Recent analysis on $h_{1}^{\perp q}$ has been given in Ref. [17].

However, no experimental investigation to extract the gluon Boer-Mulders function $\left(h_{1}^{\perp g}\right)$ has been carried out until date. Hence, the quantification of $h_{1}^{\perp g}$ is still enigmatic except knowing its theoretical upper bound [18]. Towards this, numerous proposals have been introduced to investigate $h_{1}^{\perp g}$, theoretically. The diphoton production has been suggested to probe the linearly polarized gluons in $p p \rightarrow \gamma \gamma+X$ at relativistic heavy ion collision (RHIC) [19]. Additionally, SIDIS and hadronic collision [20] processes were also proposed to probe $h_{1}^{\perp g}$ in heavy quark and dijet production respectively. It has been shown that $h_{1}^{\perp g}$ causes the imbalance of dijet in hadronic collision [21] through which an estimation of the average intrinsic transverse momentum of the partons can be obtained. It was observed that Higgs boson transverse momentum distribution at LHC has been modified by the presence of linearly polarized gluons in the unpolarized hadrons $[22,23,24]$. The modified transverse momentum spectrum provides a way to determine whether the Higgs is a pseudo-scalar or scalar [23]. The effect of linearly polarized gluons on the transverse momentum distribution in heavy quark pair production $\left(\eta_{c, b}, \chi_{c 0, b 0}\right.$ and $\left.\chi_{c 2, b 2}\right)$ has been studied in $p p$ collision [25] using non-relativistic QCD (NRQCD).

TMD factorization framework for SIDIS and DY processes has now been derived [1]. TMD functions depend on the intrinsic transverse motion $k_{\perp}$ along with the longitudinal momentum fraction $x$ of the partons whereas in collinear factorization the parton distribution functions (pdfs) depend only on $x$ in which the transverse momentum is integrated out. In addition, 
these depend on the momentum scale $Q$. The transverse momentum $\left(P_{T}\right)$ spectrum of a particular final state hadron in the scattering process seems to have Gaussian distribution [2]. This prompted the assumption that TMD pdfs exhibit Gaussian shape. A simple Gaussian model in which the TMD pdf factorizes into exponential factor function of only $k_{\perp}$ with Gaussian width $\left\langle k_{\perp}^{2}\right\rangle$ and collinear pdf which is a function of both $x$ and probing scale $Q$, seems to describe the experimental data (E288) of DY process [26] as shown in Ref. [2]. The evolution of $Q^{2}$ is taken only in collinear pdf which is known as Dokshitzer-Gribov-Lipatov-Altarelli-Parisi (DGLAP) or collinear evolution. However, Gaussian model was proven to be unsuccessful to explain the high $P_{T}$ data of $\mathrm{Z}$ boson production in DY process at CDF [27] [2]. Moreover, Gaussian width depends on the energy of the experiment to describe $P_{T}$ distribution of high energy data [2]. In order to explain the high $P_{T}$ data one must look beyond DGLAP evolution. As mentioned before, TMD factorization framework endows us evolution of TMDs [1]. By implementing the evolution of TMDs, Ref. [3] has shown that u quark TMD pdf has suppression at low $k_{\perp}$ and broad tail at high $k_{\perp}$ values. The evolution of TMDs describes the high $P_{T}$ spectrum of $\mathrm{Z}$ and $\mathrm{W}$ boson productions perfectly [28]. It is very useful to study the evolution effect on $P_{T}$ distribution because the present as well as future experiments do operate at different energies. In this work, the framework of Ref. [29] is used to implement TMD evolution.

In the recent past, a great attention has been paid to study charmonium and bottomonium productions as they provide QCD of the formation of bound state. Here we introduce a clean promising process to estimate the unpolarized gluon TMD $f_{1}^{g}\left(x, k_{\perp}\right)$ and linearly polarized gluon distribution $h_{1}^{\perp g}\left(x, k_{\perp}\right)$ in $J / \psi$ and $\Upsilon$ production in unpolarized proton-proton collision i.e., pp $\rightarrow Q \bar{Q}+X$ in the framework of transverse momentum dependent factorization. The partonic subprocesses for charmonium and bottomonium production are the two gluon fusion process $g g \rightarrow Q \bar{Q}$ and $q \bar{q} \rightarrow Q \bar{Q}$, at leading order (LO).

Among existing three models for charmonium and bottomonium production, the first one is the color singlet model (CSM) [30]. In CSM, the cross section for heavy quarkonium production is factorized similar to QCD factorization theorem. The production process can be decomposed into two steps. The first step is creation of on-shell heavy quark pair which is calculated perturbatively. The other step is the binding of the $Q \bar{Q}$ pair into physical color singlet state which is encoded in the long distance factor, the wave function. Generally the wave function is obtained 
by fitting data or from potential models. CSM suggests that spin and color quantum numbers of $Q \bar{Q}$ pair do not alter during hadronization process. Therefore, to produce a physical color singlet state it requires that the $Q \bar{Q}$ pair should be in color singlet state. Thus the name CSM for this model. Nevertheless, CSM is unable to describe the large transverse momentum of $J / \psi$, $\Upsilon$ and $\psi(2 s)[31,32]$.

The second one is NRQCD model [33] in which the cross section is factorized just like CSM. The quarkonium cross section is a product of short and long distance factors summed over all possible color, spin and angular momentum quantum numbers of the $Q \bar{Q}$ pair. In this model the formation of heavy quark pair is either in color octet or color singlet state. The short distance factor can be calculated with appropriate quantum numbers using perturbation theory. The long distance factor, the nonperturbative matrix element, describes the transition probability of the $Q \bar{Q}$ pair from colored state to colorless physical state, which can be expanded in powers of $v$ where $v$ is the relative velocity of the heavy quark in the quarkonium rest frame. The values are $v^{2}=0.1$ and 0.3 for bottomonium and charmonium respectively.

The third model is the color evaporation model (CEM), which was first developed in 1977 by F.Halzen, Matsuda [34] and Fritsch [35]. In CEM, it is assumed that the heavy quark pair is produced perturbatively with definite spin and color quantum numbers which can be calculated upto a desired order in $\alpha_{s}$. Thereafter, the heavy quark pair radiates soft gluons to evolve into any physical color neutral quarkonium state with quantum numbers different than that of initial heavy quark pair. The process of hadronization of the quarkonium from heavy quark pair is usually referred to as nonperturbative process. The CEM acquired the name "color evaporation", since the color of the initial $Q \bar{Q}$ pair does not affect the final quarkonium state. According to CEM, the cross section of quarkonium state is some long distance factor times the cross section of the $Q \bar{Q}$ pair with invariant mass below the threshold mass. Typically, the long distance factors are considered to be universal which are determined by fitting the heavy quark pair cross section with experimental data. Schuler and R. Vogt [36] determined the long distance factors for $J / \psi$ and $\Upsilon$ to be 0.055 and 0.087 respectively. Amundson et al, [37] introduced another constraint in CEM, namely the probability of producing a color singlet quarkonium state is only $1 / 9$ of the heavy quark pair production. The long distance factors deduced in this version of CEM for $J / \psi$ and $\Upsilon$ are 0.47 [37] and 0.62 [38] respectively. The general prediction 
of CEM is that the probability of forming any quarkonium state is independent of the color and spin quantum numbers. This model is found to be in close match with the experimental data with an inclusion of a phenomenological factor in the cross section that is dependent on the Gaussian distribution of the transverse momentum of the quarkonium [39]. Given an importance to its simplicity, the present work employs the CEM to advance the understanding of the TMDs and their evolution. As a whole, the paper contains five sections including introduction. Section II presents the formalism for $J / \psi$ and $\Upsilon$ production. The TMD evolution formalism is presented in III. Numerical results are presented in the Section IV along with the conclusion in Section $\mathrm{V}$.

\section{CHARMONIUM $(J / \psi)$ AND BOTTOMONIUM $(\Upsilon)$ PRODUCTION}

The formalism for charmonium and bottomonium production in CEM is explained as follows. The cross section for this production is proportional to the rate of production of $Q \bar{Q}$ pair which is integrated over the mass values ranging from $2 m_{Q}$ to $2 m_{Q \bar{q}}$ [40], see also [41, 42]

$$
\sigma=\frac{\rho}{9} \int_{2 m_{Q}}^{2 m_{Q \bar{q}}} d M \frac{d \sigma_{Q \bar{Q}}}{d M}
$$

where $m_{Q}$ is the mass of charm or bottom quark and $m_{Q \bar{q}}$ is the mass of lightest $\mathrm{D}$ meson for

charmonium and B meson for bottomonium. $\frac{d \sigma_{Q \bar{Q}}}{d M}$ can be calculated perturbatively and $M$ is the invariant mass of the $Q \bar{Q}$ pair. The probability of producing the quarkonium is zero if the invariant mass of heavy quark pair is more than $2 m_{Q \bar{q}}$. Here $\rho$ is long distance factor and we have taken 0.47 [37] and 0.62 [38] for production of $J / \psi$ and $\Upsilon$ respectively.

We consider the following process (unpolarized proton-proton collision) for charmonium and bottomonium production

$$
h\left(P_{A}\right)+h\left(P_{B}\right) \rightarrow Q \bar{Q}(q)+X
$$

where the four momenta are given within round brackets. We choose the frame in which the proton $A$ is moving along $+\hat{z}$ axis and proton $B$ is moving along $-\hat{z}$ axis in the center of mass (c.m) frame with four momenta $P_{A}^{\mu}=\frac{\sqrt{s}}{2}(1,0,0,1)$ and $P_{B}^{\mu}=\frac{\sqrt{s}}{2}(1,0,0,-1)$ respectively. The $Q \bar{Q}$ pair is produced from two gluon fusion and $q \bar{q}$ annihilation partonic subprocesses, which 
are shown by Feynman diagrams in Fig.1. The production of the quarkonium is important only in the low transverse momentum region of the produced charmonium and bottomonium.
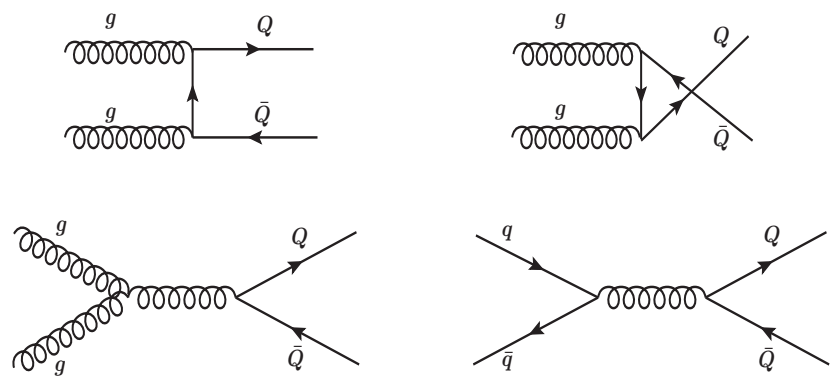

FIG. 1: Feynman diagrams for gluon-gluon fusion and $q \bar{q}$ annihilation processes at leading order.

The assumption that the TMD factorization holds good at sufficiently high energies is adopted in similar lines with Ref. [4, 43]. The cross section using generalized CEM and TMD factorization formalism for Eq.(2) is

$$
\begin{aligned}
d \sigma= & \frac{\rho}{9} \int d x_{a} d x_{b} d^{2} \mathbf{k}_{\perp a} d^{2} \mathbf{k}_{\perp b}\left\{\Phi_{g}^{\mu \nu}\left(x_{a}, \mathbf{k}_{\perp a}\right) \Phi_{g \mu \nu}\left(x_{b}, \mathbf{k}_{\perp b}\right) d \hat{\sigma}^{g g \rightarrow Q \bar{Q}}+\right. \\
& {\left.\left[\Phi^{q}\left(x_{a}, \mathbf{k}_{\perp a}^{2}\right) \Phi^{\bar{q}}\left(x_{b}, \mathbf{k}_{\perp b}^{2}\right)+\Phi^{\bar{q}}\left(x_{a}, \mathbf{k}_{\perp a}^{2}\right) \Phi^{q}\left(x_{b}, \mathbf{k}_{\perp b}^{2}\right)\right] d \hat{\sigma}^{q \bar{q} \rightarrow Q \bar{Q}}\right\}, }
\end{aligned}
$$

where $x_{a}, x_{b}$ are the longitudinal momentum fractions and $k_{\perp a}, k_{\perp b}$ are the transverse momentum of the incoming gluons and quarks. Here, $\mathrm{q}=\mathrm{u}, \overline{\mathrm{u}}, \mathrm{d}, \overline{\mathrm{d}}, s, \bar{s}$ and $Q=\mathrm{c}$ or $\mathrm{b}$, depending on whether $J / \psi$ or $\Upsilon$ is produced. The gluon field strengths $F^{\mu \nu}(0)$ and $F^{\mu \nu}(\lambda)$ that are evaluated at fixed light front time $\lambda^{+}=\lambda . n=0$ are used to define the gluon correlator (omitting gauge link) $\Phi_{g}^{\mu \nu}$. Here, $n$ is light-like vector and is conjugate to the proton four momentum $P$. At leading twist, the gluon correlator of unpolarized hadron contains two TMD gluon distribution functions [5]

$$
\begin{aligned}
\Phi_{g}^{\mu \nu}\left(x, \mathbf{k}_{\perp}\right) & =\left.\frac{n_{\rho} n_{\sigma}}{(k \cdot n)^{2}} \int \frac{d(\lambda . P) d^{2} \lambda_{T}}{(2 \pi)^{3}} e^{i k . \lambda}\left\langle P\left|\operatorname{Tr}\left[F^{\mu \nu}(0) F^{\mu \nu}(\lambda)\right]\right| P\right\rangle\right|_{L F} \\
& =-\frac{1}{2 x}\left\{g_{T}^{\mu \nu} f_{1}^{g}\left(x, \mathbf{k}_{\perp}^{2}\right)-\left(\frac{k_{\perp}^{\mu} k_{\perp}^{\nu}}{M_{h}^{2}}+g_{T}^{\mu \nu} \frac{\mathbf{k}_{\perp}^{2}}{2 M_{h}^{2}}\right) h_{1}^{\perp g}\left(x, \mathbf{k}_{\perp}^{2}\right)\right\} .
\end{aligned}
$$


The quark correlator with omitting the gauge link is defined as [20]

$$
\begin{aligned}
\Phi^{q}\left(x, \mathbf{k}_{\perp}\right) & \left.=\int \frac{d(\lambda . P) d^{2} \lambda_{T}}{(2 \pi)^{3}} e^{i k . \lambda}\langle P| \bar{\psi}(0) \psi(\lambda)\right]\left.|P\rangle\right|_{L F} \\
& =\frac{1}{2}\left\{f_{1}^{q}\left(x, \mathbf{k}_{\perp}^{2}\right) \not P+i h_{1}^{\perp q}\left(x, \mathbf{k}_{\perp}^{2}\right) \frac{\left[k_{\perp}, \not P\right]}{2 M_{h}}\right\},
\end{aligned}
$$

and the antiquark correlator is given by [20]

$$
\begin{aligned}
\Phi^{\bar{q}}\left(x, \mathbf{k}_{\perp}\right) & \left.=-\int \frac{d(\lambda . P) d^{2} \lambda_{T}}{(2 \pi)^{3}} e^{-i k \cdot \lambda}\langle P| \bar{\psi}(0) \psi(\lambda)\right]\left.|P\rangle\right|_{L F} \\
& =\frac{1}{2}\left\{f_{1}^{\bar{q}}\left(x, \mathbf{k}_{\perp}^{2}\right) \not P+i h_{1}^{\perp \bar{q}}\left(x, \mathbf{k}_{\perp}^{2}\right) \frac{\left[k_{\perp}, \not P\right]}{2 M_{h}}\right\} .
\end{aligned}
$$

Here $k_{\perp}^{2}=-\mathbf{k}_{\perp}^{2}, g_{T}^{\mu \nu}=g^{\mu \nu}-P^{\mu} n^{\nu} / P . n-n^{\mu} P^{\nu} / P . n$ and $M_{h}$ is the mass of hadron. The unpolarized and the linearly polarized gluon distribution functions are respectively denoted by $f_{1}^{g}\left(x, \mathbf{k}_{\perp}^{2}\right)$ and $h_{1}^{\perp g}\left(x, \mathbf{k}_{\perp}^{2}\right)$. Similarly, $f_{1}^{q, \bar{q}}\left(x, \mathbf{k}_{\perp}^{2}\right)$ and $h_{1}^{\perp q, \bar{q}}\left(x, \mathbf{k}_{\perp}^{2}\right)$ represent the distribution of unpolarized and transversely polarized quark (antiquark) respectively.

The $d \hat{\sigma}$, the partonic cross section for $g g \rightarrow Q \bar{Q}$ and $q \bar{q} \rightarrow Q \bar{Q}$, is given by

$$
d \hat{\sigma}^{g g, q \bar{q} \rightarrow Q \bar{Q}}=\frac{1}{2 \hat{s}} \frac{d^{3} p_{Q}}{2 E_{Q}} \frac{d^{3} p_{\bar{Q}}}{2 E_{\bar{Q}}} \frac{1}{(2 \pi)^{2}} \delta^{4}\left(p_{a}+p_{b}-p_{Q}-p_{\bar{Q}}\right) \overline{\left|M_{g g, q \bar{q} \rightarrow Q \bar{Q}}\right|^{2}},
$$

where $p_{a}$ and $p_{b}$ are the four momentum vectors of incoming gluons and quarks and $p_{Q}\left(p_{\bar{Q}}\right)$ is the produced quark (antiquark) four momentum. Let us define a four momentum vector $q=\left(q_{0}, \mathbf{q}_{T}, q_{L}\right)$ of the $Q \bar{Q}$ pair, where $q_{0}, q_{L}$ and $\mathbf{q}_{T}$ are the energy, longitudinal and transverse components respectively. The four momentum of quarkonium pair is $q=p_{Q}+p_{\bar{Q}}$ and using this relation one can rewrite $\frac{d^{3} p_{\bar{Q}}}{2 E_{\bar{Q}}}=d^{4} p_{\bar{Q}} \delta\left(p_{\bar{Q}}^{2}-m_{\bar{Q}}^{2}\right)$. By changing the variables $p_{\bar{Q}}$ and $q_{0}$ and $q_{L}$ to $q$ and $M^{2}$ and $y$ (rapidity) respectively [44], we get

$$
\begin{aligned}
\frac{d^{3} p_{\bar{Q}}}{2 E_{\bar{Q}}} & =d^{4} q \delta\left(\left(q-p_{Q}\right)^{2}-m_{Q}^{2}\right), \\
d M^{2} d y & =2 d q_{0} d q_{L} .
\end{aligned}
$$

The partonic cross section can be written as

$$
\hat{\sigma}^{g g, q \bar{q} \rightarrow Q \bar{Q}}=\frac{1}{2 M^{2}} \int \frac{d^{3} p_{Q}}{2 E_{Q}} \delta\left(\left(q-p_{Q}\right)^{2}-m_{Q}^{2}\right) \overline{\left|M_{g g, q \bar{q} \rightarrow Q \bar{Q}}\right|^{2}} .
$$


The differential cross section Eq.(3) can be written as functions of transverse momentum, rapidity and squared mass of quarkonium using Eq.(8) and (9)

$$
\begin{aligned}
\frac{d^{4} \sigma}{d y d M^{2} d^{2} \mathbf{q}_{T}}= & \frac{\rho}{18} \int d x_{a} d x_{b} d^{2} \mathbf{k}_{\perp a} d^{2} \mathbf{k}_{\perp b} \delta^{4}\left(p_{a}+p_{b}-q\right)\left\{\Phi_{g}^{\mu \nu}\left(x_{a}, \mathbf{k}_{\perp a}\right) \Phi_{g \mu \nu}\left(x_{b}, \mathbf{k}_{\perp b}\right) \hat{\sigma}^{g g \rightarrow Q \bar{Q}}+\right. \\
& {\left.\left[\Phi^{q}\left(x_{a}, \mathbf{k}_{\perp a}^{2}\right) \Phi^{\bar{q}}\left(x_{b}, \mathbf{k}_{\perp b}^{2}\right)+\Phi^{\bar{q}}\left(x_{a}, \mathbf{k}_{\perp a}^{2}\right) \Phi^{q}\left(x_{b}, \mathbf{k}_{\perp b}^{2}\right)\right] \hat{\sigma}^{q \bar{q} \rightarrow Q \bar{Q}}\right\} . }
\end{aligned}
$$

To obtain the differential cross section in terms of TMD distribution functions, we substitute Eq.(4), (5) and (6) in Eq.(10)

$$
\begin{aligned}
\frac{d^{4} \sigma}{d y d M^{2} d^{2} \mathbf{q}_{T}}= & \frac{\rho}{18} \int d x_{a} d x_{b} d^{2} \mathbf{k}_{\perp a} d^{2} \mathbf{k}_{\perp b} \delta^{4}\left(p_{a}+p_{b}-q\right)\left\{\frac{1}{2 x_{a} x_{b}}\right. \\
& \times\left[f_{1}^{g}\left(x_{a}, \mathbf{k}_{\perp a}^{2}\right) f_{1}^{g}\left(x_{b}, \mathbf{k}_{\perp b}^{2}\right)+w h_{1}^{\perp g}\left(x_{a}, \mathbf{k}_{\perp a}^{2}\right) h_{1}^{\perp g}\left(x_{b}, \mathbf{k}_{\perp b}^{2}\right)\right] \hat{\sigma}^{g g \rightarrow Q \bar{Q}}\left(M^{2}\right) \\
& \left.+\frac{1}{4} \sum_{\mathrm{q}}\left[f_{1}^{q}\left(x_{a}, \mathbf{k}_{\perp a}^{2}\right) f_{1}^{\bar{q}}\left(x_{b}, \mathbf{k}_{\perp b}^{2}\right)+f_{1}^{\bar{q}}\left(x_{a}, \mathbf{k}_{\perp a}^{2}\right) f_{1}^{q}\left(x_{b}, \mathbf{k}_{\perp b}^{2}\right)\right] \hat{\sigma}^{q \bar{q} \rightarrow Q \bar{Q}}\left(M^{2}\right)\right\},
\end{aligned}
$$

where $w$ is weight factor of the transverse momentum

$$
w=\frac{1}{2 M_{h}^{4}}\left[\left(\mathbf{k}_{\perp a} \cdot \mathbf{k}_{\perp b}\right)^{2}-\frac{1}{2} \mathbf{k}_{\perp a}^{2} \mathbf{k}_{\perp b}^{2}\right] .
$$

In most parts of this study, the transversely polarized quark and antiquark contributions have been neglected since the gluon channel is dominant, as shown later. The total partonic cross sections $\hat{\sigma}$ are calculated perturbatively [45]

$$
\begin{aligned}
\hat{\sigma}^{g g \rightarrow Q \bar{Q}} & =\frac{\pi \alpha_{s}^{2}}{3 M^{2}}\left[\left(1+\gamma+\frac{1}{16} \gamma^{2}\right) \ln \frac{1+\sqrt{1-\gamma}}{1-\sqrt{1-\gamma}}-\left(\frac{7}{4}+\frac{31}{16} \gamma\right) \sqrt{1-\gamma}\right] \\
\hat{\sigma}^{q \bar{q} \rightarrow Q \bar{Q}} & =\frac{2}{9}\left(\frac{4 \pi \alpha_{s}^{2}}{3 M^{2}}\right)\left(1+\frac{1}{2} \gamma\right) \sqrt{1-\gamma}
\end{aligned}
$$

where $\gamma=\frac{4 m_{Q}^{2}}{M^{2}}$ and $M^{2}=\hat{s}, \sqrt{\hat{s}}$ is the center-of-mass energy of the partonic subprocess. In line with Ref. [44], the four momentum conservation $\delta$ function can also be written as

$$
\begin{aligned}
\delta^{4}\left(p_{a}+p_{b}-q\right) & =\delta\left(E_{a}+E_{b}-q_{0}\right) \delta\left(p_{z a}+p_{z b}-q_{L}\right) \delta^{2}\left(\mathbf{k}_{\perp a}+\mathbf{k}_{\perp b}-\mathbf{q}_{T}\right) \\
& =\frac{2}{s} \delta\left(x_{a}-\frac{M e^{y}}{\sqrt{s}}\right) \delta\left(x_{b}-\frac{M e^{-y}}{\sqrt{s}}\right) \delta^{2}\left(\mathbf{k}_{\perp a}+\mathbf{k}_{\perp b}-\mathbf{q}_{T}\right) .
\end{aligned}
$$


After performing integrations over $x_{a}$ and $x_{b}$, the two $\delta$ functions in Eq.(16) gives

$$
x_{a, b}=\frac{M}{\sqrt{s}} e^{ \pm y} .
$$

$\sqrt{s}$ is the center-of-mass energy. The expression for the cross section obtained is as follows

$$
\begin{aligned}
\frac{d^{4} \sigma}{d y d M^{2} d^{2} \mathbf{q}_{T}}= & \frac{\rho}{9 s} \int d^{2} \mathbf{k}_{\perp a} d^{2} \mathbf{k}_{\perp b} \delta^{2}\left(\mathbf{k}_{\perp a}+\mathbf{k}_{\perp b}-\mathbf{q}_{T}\right)\left\{\frac { 1 } { 2 } \left[f_{1}^{g}\left(x_{a}, \mathbf{k}_{\perp a}^{2}\right) f_{1}^{g}\left(x_{b}, \mathbf{k}_{\perp b}^{2}\right)\right.\right. \\
& \left.+w h_{1}^{\perp g}\left(x_{a}, \mathbf{k}_{\perp a}^{2}\right) h_{1}^{\perp g}\left(x_{b}, \mathbf{k}_{\perp b}^{2}\right)\right] \hat{\sigma}^{g g \rightarrow Q \bar{Q}}\left(M^{2}\right) \\
& \left.+\frac{1}{4} \sum_{\mathbf{q}}\left[f_{1}^{q}\left(x_{a}, \mathbf{k}_{\perp a}^{2}\right) f_{1}^{\bar{q}}\left(x_{b}, \mathbf{k}_{\perp b}^{2}\right)+f_{1}^{\bar{q}}\left(x_{a}, \mathbf{k}_{\perp a}^{2}\right) f_{1}^{q}\left(x_{b}, \mathbf{k}_{\perp b}^{2}\right)\right] \hat{\sigma}^{q \bar{q} \rightarrow Q \bar{Q}}\left(M^{2}\right)\right\} .
\end{aligned}
$$

We can eliminate $\mathbf{k}_{\perp b}$ by integrating and finally we reach

$$
\frac{d^{2} \sigma^{f f+h h}}{d y d q_{T}^{2}}=\frac{d^{2} \sigma^{f f}}{d y d q_{T}^{2}}+\frac{d^{2} \sigma^{h h}}{d y d q_{T}^{2}},
$$

where

$$
\begin{aligned}
\frac{d^{2} \sigma^{f f}}{d y d q_{T}^{2}}= & \frac{\rho}{36 s} \int d M^{2} \int d \phi_{q_{T}} \int d k_{\perp a} k_{\perp a} \int d \phi_{k_{\perp a}}\left\{f_{1}^{g}\left(x_{a}, \mathbf{k}_{\perp a}^{2}\right)\right. \\
& \times f_{1}^{g}\left(x_{b},\left(\mathbf{q}_{T}-\mathbf{k}_{\perp a}\right)^{2}\right) \hat{\sigma}^{g g \rightarrow Q \bar{Q}}\left(M^{2}\right)+\frac{1}{2} \sum_{q}\left[f_{1}^{q}\left(x_{a}, \mathbf{k}_{\perp a}^{2}\right) f_{1}^{\bar{q}}\left(x_{b},\left(\mathbf{q}_{T}-\mathbf{k}_{\perp a}\right)^{2}\right)\right. \\
& \left.\left.+f_{1}^{\bar{q}}\left(x_{a}, \mathbf{k}_{\perp a}^{2}\right) f_{1}^{q}\left(x_{b},\left(\mathbf{q}_{T}-\mathbf{k}_{\perp a}\right)^{2}\right)\right] \hat{\sigma}^{q \bar{q} \rightarrow Q \bar{Q}}\left(M^{2}\right)\right\},
\end{aligned}
$$

and

$$
\begin{aligned}
\frac{d^{2} \sigma^{h h}}{d y d q_{T}^{2}}= & \frac{\rho}{36 s} \frac{1}{2 M_{h}^{4}} \int d M^{2} \int d \phi_{q_{T}} \int d k_{\perp a} k_{\perp a} \int d \phi_{k_{\perp a}} \\
& \times\left[\frac{1}{2} k_{\perp a}^{4}-\frac{1}{2} k_{\perp a}^{2} q_{T}^{2}-q_{T} k_{\perp a}^{3} \cos \left(\phi_{k_{\perp a}}-\phi_{q_{T}}\right)+q_{T}^{2} k_{\perp a}^{2} \cos ^{2}\left(\phi_{k_{\perp a}}-\phi_{q_{T}}\right)\right] \\
& \times h_{1}^{\perp g}\left(x_{a}, \mathbf{k}_{\perp a}^{2}\right) h_{1}^{\perp g}\left(x_{b},\left(\mathbf{q}_{T}-\mathbf{k}_{\perp a}\right)^{2}\right) \hat{\sigma}^{g g \rightarrow Q \bar{Q}}\left(M^{2}\right) .
\end{aligned}
$$

Here, $\phi_{k_{\perp a}}$ is the azimuthal angle of gluon and quark and the azimuthal angle of the quarko$\operatorname{nium}$ is $\phi_{q_{T}}$.

\section{TMD EVOLUTION}

In the present section, we discuss the model used for the TMDs as well as the TMD evolution. As per the general conception, we assume that unpolarized distribution functions of glu- 
ons, quarks and antiquarks TMDs do simply depend on Gaussian form of gluon's and quark's transverse momentum [46]

$$
f_{1}^{g, q}\left(x, \mathbf{k}_{\perp}^{2}\right)=f_{1}^{g, q}\left(x, Q^{2}\right) \frac{1}{\pi\left\langle k_{\perp}^{2}\right\rangle} e^{-\mathbf{k}_{\perp}^{2} /\left\langle k_{\perp}^{2}\right\rangle} .
$$

Here, TMD pdf is factorized into $k_{\perp}$ and $x$ dependencies. $Q^{2}$ dependence is only in $f_{1}^{g, q}\left(x, Q^{2}\right)$ which is the usual collinear pdf evaluated at scale $Q^{2}$. We have chosen $Q^{2}=M^{2}$ that is known as collinear or DGLAP evolution. The factorized form of $h_{1}^{\perp g}$ [25] is given by

$$
h_{1}^{\perp g}\left(x, \mathbf{k}_{\perp}^{2}\right)=\frac{M_{h}^{2} f_{1}^{g}\left(x, Q^{2}\right)}{\pi\left\langle k_{\perp}^{2}\right\rangle^{2}} \frac{2(1-r)}{r} e^{1-\mathbf{k}_{\perp}^{2} \frac{1}{r\left\langle k_{\perp}^{2}\right\rangle},}
$$

where $r$ is the parameter which has the range $0<r<1$. The Eq.(23) obeys the model independent positive bound [18] for all values of $x$ and $k_{\perp}$

$$
\frac{\mathbf{k}_{\perp}^{2}}{2 M_{h}^{2}}\left|h_{1}^{\perp g}\left(x, \mathbf{k}_{\perp}^{2}\right)\right| \leq f_{1}^{g}\left(x, \mathbf{k}_{\perp}^{2}\right) .
$$

In this work, we use two values for squared intrinsic average transverse momentum of gluons and quarks i.e., $\left\langle k_{\perp}^{2}\right\rangle=0.25 \mathrm{GeV}^{2}$ and $1 \mathrm{GeV}^{2}$ [25]. The parameter values chosen are $r=\frac{1}{3}$ and $\frac{2}{3}[25]$.

\section{A. Model-I}

In model-I, we do not use an upper limit of the transverse momentum integration. The exponential behavior of unpolarized and linearly polarized TMDs allows us to integrate analytically with respect to $k_{\perp a}$ and we obtain

$$
\begin{aligned}
\frac{d^{2} \sigma^{f f}}{d y d q_{T}^{2}} & =\frac{\beta \rho}{36 s} \int_{4 m_{Q}^{2}}^{4 m_{Q \bar{q}}^{2}} d M^{2} e^{-q_{T}^{2} \frac{\beta}{2}}\left\{f_{1}^{g}\left(x_{a}\right) f_{1}^{g}\left(x_{b}\right) \hat{\sigma}^{g g \rightarrow Q \bar{Q}}\left(M^{2}\right)\right. \\
& \left.+\frac{1}{2} \sum_{q}\left[f_{1}^{q}\left(x_{a}\right) f_{1}^{\bar{q}}\left(x_{b}\right)+f_{1}^{\bar{q}}\left(x_{a}\right) f_{1}^{q}\left(x_{b}\right)\right] \hat{\sigma}^{q \bar{q} \rightarrow Q \bar{Q}}\left(M^{2}\right)\right\},
\end{aligned}
$$

and

$$
\begin{array}{r}
\frac{d^{2} \sigma^{h h}}{d y d q_{T}^{2}}=\frac{\beta \rho r(1-r)^{2}}{72 s} \int_{4 m_{Q}^{2}}^{4 m_{Q \bar{q}}^{2}} d M^{2}\left[1-\frac{\beta q_{T}^{2}}{r}+\frac{\beta^{2} q_{T}^{4}}{8 r^{2}}\right] e^{\left[2-\frac{\beta}{2 r} q_{T}^{2}\right]} f_{1}^{g}\left(x_{a}\right) f_{1}^{g}\left(x_{b}\right) \\
\times \hat{\sigma}^{g g \rightarrow Q \bar{Q}}\left(M^{2}\right),
\end{array}
$$

where $\beta=\frac{1}{\left\langle k_{\perp a}^{2}\right\rangle}=\frac{1}{\left\langle\left(q_{T}-k_{\perp a}\right)^{2}\right\rangle}$. 


\section{B. Model-II}

In this model, we consider the effective intrinsic motion of the gluons for Gaussian distribution to be restricted to $k_{\max }=\sqrt{\left\langle k_{\perp a}^{2}\right\rangle}$ [47]. Hence the expressions for quarkonium production are given by

$$
\begin{aligned}
\frac{d^{2} \sigma^{f f}}{d y d q_{T}^{2}}= & \frac{\beta^{2} \rho}{36 s \pi^{2}} \int_{4 m_{Q}^{2}}^{4 m_{Q \bar{q}}^{2}} d M^{2} \int d \phi_{q_{T}} \int_{0}^{k_{\max }} d k_{\perp a} k_{\perp a} \int d \phi_{k_{\perp a}} e^{-\Delta \beta} \\
& \times\left\{f_{1}^{g}\left(x_{a}\right) f_{1}^{g}\left(x_{b}\right) \hat{\sigma}^{g g \rightarrow Q \bar{Q}}\left(M^{2}\right)+\frac{1}{2} \sum_{q}\left[f_{1}^{q}\left(x_{a}\right) f_{1}^{\bar{q}}\left(x_{b}\right)+f_{1}^{\bar{q}}\left(x_{a}\right) f_{1}^{q}\left(x_{b}\right)\right]\right. \\
& \left.\times \hat{\sigma}^{q \bar{q} \rightarrow Q \bar{Q}}\left(M^{2}\right)\right\},
\end{aligned}
$$

and

$$
\begin{aligned}
\frac{d^{2} \sigma^{h h}}{d y d q_{T}^{2}}= & \frac{\beta^{4} \rho(1-r)^{2}}{18 s r^{2} \pi^{2}} \int_{4 m_{Q}^{2}}^{4 m_{Q \bar{q}}^{2}} d M^{2} \int d \phi_{q_{T}} \int_{0}^{k_{\max }} d k_{\perp a} k_{\perp a} \int d \phi_{k_{\perp a}} \\
\times & {\left[\frac{1}{2} k_{\perp a}^{4}-\frac{1}{2} k_{\perp a}^{2} q_{T}^{2}-q_{T} k_{\perp a}^{3} \cos \left(\phi_{k_{\perp a}}-\phi_{q_{T}}\right)+q_{T}^{2} k_{\perp a}^{2} \cos ^{2}\left(\phi_{k_{\perp a}}-\phi_{q_{T}}\right)\right] } \\
& \times e^{\left[2-\frac{\beta}{r} \Delta\right]} f_{1}^{g}\left(x_{a}\right) f_{1}^{g}\left(x_{b}\right) \hat{\sigma}^{g g \rightarrow Q \bar{Q}}\left(M^{2}\right),
\end{aligned}
$$

where $\Delta=2 k_{\perp a}^{2}+q_{T}^{2}-2 q_{T} k_{\perp a} \cos \left(\phi_{k_{\perp a}}-\phi_{q_{T}}\right)$.

For the evolution of TMDs we adopted the formalism Ref. [29] as mentioned in the introduction. The TMD evolution formalism has been formulated in two dimensional coordinate space $\left(b_{\perp}\right.$-space). Therefore, transverse momentum dependent gluon-gluon correlator function is Fourier transformed into $b_{\perp}$-space which is defined as

$$
\Phi\left(x, \mathbf{b}_{\perp}\right)=\int d^{2} \mathbf{k}_{\perp} e^{-i \mathbf{k}_{\perp} \cdot \mathbf{b}_{\perp}} \Phi\left(x, \mathbf{k}_{\perp}\right),
$$

and the inverse Fourier transformation is

$$
\Phi\left(x, \mathbf{k}_{\perp}\right)=\frac{1}{(2 \pi)^{2}} \int d^{2} \mathbf{b}_{\perp} e^{i \mathbf{k}_{\perp} \cdot \mathbf{b}_{\perp}} \Phi\left(x, \mathbf{b}_{\perp}\right) .
$$

After performing delta function integrations in Eq.(10), the differential cross section of quarkonium can be written as following

$$
\frac{d^{4} \sigma}{d y d M^{2} d^{2} \mathbf{q}_{T}}=\frac{\rho}{9 s} \int d^{2} \mathbf{k}_{\perp a} \Phi_{g}^{\mu \nu}\left(x_{a}, \mathbf{k}_{\perp a}\right) \Phi_{g \mu \nu}\left(x_{b}, \mathbf{q}_{T}-\mathbf{k}_{\perp a}\right) \hat{\sigma}^{g g \rightarrow Q \bar{Q}} .
$$


In the evolution of TMDs, only TMD pdfs of gluons are considered. Quark contribution is neglected because of its insignificance in the quarkonium production as depicted in Fig. 3. Substituting Eq.(29) and (30) in (31), one can obtain the following differential cross section in $b_{\perp}$-space as,

$$
\frac{d^{4} \sigma}{d y d M^{2} d^{2} \mathbf{q}_{T}}=\frac{\rho}{9 s} \frac{1}{(2 \pi)^{2}} \int d^{2} \mathbf{b}_{\perp} e^{i \mathbf{q}_{T} \cdot \mathbf{b}_{\perp}} \Phi_{g}^{\mu \nu}\left(x_{a}, \mathbf{b}_{\perp}\right) \Phi_{g \mu \nu}\left(x_{b}, \mathbf{b}_{\perp}\right) \hat{\sigma}^{g g \rightarrow Q \bar{Q}} .
$$

In lines of Ref. [29], the gluon correlator function in $b_{\perp}$-space is given by

$$
\Phi^{g}\left(x, \mathbf{b}_{\perp}\right)=\frac{1}{2 x}\left\{g_{T}^{\mu \nu} f_{1}^{g}\left(x, \mathbf{b}_{\perp}^{2}\right)-\left(\frac{2 b_{\perp}^{\mu} b_{\perp}^{\nu}}{b_{\perp}^{2}}-g_{T}^{\mu \nu}\right) h_{1}^{\perp g}\left(x, \mathbf{b}_{\perp}^{2}\right)\right\} .
$$

The differential cross section of quarkonium in terms of $b_{\perp}$-space pdfs is obtained by inserting Eq.(33) in Eq.(32)

$$
\begin{aligned}
\frac{d^{4} \sigma}{d y d M^{2} d^{2} \mathbf{q}_{T}}= & \frac{\rho}{18 s} \frac{1}{2 \pi} \int_{0}^{\infty} b_{\perp} d b_{\perp} J_{0}\left(q_{T} b_{\perp}\right)\left\{f_{1}^{g}\left(x_{a}, b_{\perp}^{2}\right) f_{1}^{g}\left(x_{b}, b_{\perp}^{2}\right)\right. \\
& \left.+h_{1}^{\perp g}\left(x_{a}, b_{\perp}^{2}\right) h_{1}^{\perp g}\left(x_{b}, b_{\perp}^{2}\right)\right\} \hat{\sigma}^{g g \rightarrow Q \bar{Q}}\left(M^{2}\right),
\end{aligned}
$$

where $J_{0}$ is the Bessel function of zeroth order. TMD pdfs depend not only on the renormalization scale $\mu$ but also on $\zeta$. Here, $\zeta$ is an auxiliary parameter which is introduced to regularize the light cone divergence in TMD factorization formalism [1]. Collins-Soper (CS) and Renormalization Group (RG) equations are obtained by taking evolution in $\zeta$ and $\mu$ respectively $[1,3]$. Using CS and RG equations one obtains the evolution of TMDs from initial scale $Q_{i}=c / b_{*}\left(b_{\perp}\right)$ to final scale $Q_{f}=Q[3,48]$ :

$$
f\left(x, b_{\perp}, Q_{f}, \zeta\right)=f\left(x, b_{\perp}, Q_{i}, \zeta\right) R_{\text {pert }}\left(Q_{f}, Q_{i}, b_{*}\right) R_{N P}\left(Q_{f}, Q_{i}, b_{\perp}\right)
$$

where $R_{\text {pert }}$ and $R_{N P}$ are perturbative and non-perturbative parts respectively. $c / b_{*}$ is the initial scale where $c=2 e^{-\gamma_{\epsilon}}$ bearing Euler's constant $\gamma_{\epsilon} \approx 0.577$. Here, $b_{*}\left(b_{\perp}\right)=\frac{b_{\perp}}{\sqrt{1+\left(\frac{b_{\perp}}{b_{\max }}\right)^{2}}} \approx$ $b_{\max }$ when $b_{\perp} \rightarrow \infty$ and $b_{*}\left(b_{\perp}\right) \approx b_{\perp}$ when $b_{\perp} \rightarrow 0$ is usually known as $b_{*}$ prescription. This prescription is used to separate out nonperturbative part from the evolution kernel since the evolution kernel is not valid at larger values of $b_{\perp}$ [1]. The separated nonperturbative part is embodied in the exponential containing the nonperturbative Sudakov factor, $R_{N P}$. The evolution kernel is given by [29]

$$
R_{\text {pert }}\left(Q_{f}, Q_{i}, b_{*}\right)=\exp \left\{-\int_{c / b_{*}}^{Q} \frac{d \mu}{\mu}\left(A \log \left(\frac{Q^{2}}{\mu^{2}}\right)+B\right)\right\}
$$


where $A$ and $B$ are anomalous dimensions of evolution kernel and TMD pdf respectively which have perturbative expansion like

$$
A=\sum_{n=1}^{\infty}\left(\frac{\alpha_{s}(\mu)}{\pi}\right)^{n} A_{n}
$$

and

$$
B=\sum_{n=1}^{\infty}\left(\frac{\alpha_{s}(\mu)}{\pi}\right)^{n} B_{n}
$$

The first order expansion coefficients in $\alpha_{s}$ are $A_{1}=C_{A}$ and $B_{1}=-\frac{1}{2}\left(\frac{11}{3} C_{A}-\frac{2}{3} N_{f}\right)$. The anomalous dimensions are derived up to 3-loop level [49]. The perturbative Sudakov factor, in our case, is the same for unpolarized and linearly polarized gluon TMDs [28, 50]. The evolution kernel resummed up to NLL accuracy in exploration of Sivers asymmetry in SIDIS and DY processes [28]. Generally, the nonperturbative factor, $R_{N P}$, is extracted by fitting with experimental data. We choose the nonperturbative Sudakov factor as given in Aybat et al. [3] which describes the SIDIS and Z boson data with good accuracy.

$$
R_{N P}=\exp \left\{-\left[\frac{g_{2}}{2} \log \frac{Q}{2 Q_{0}}+\frac{g_{1}}{2}\left(1+2 g_{3} \log \frac{10 x x_{0}}{x_{0}+x}\right)\right] b_{\perp}^{2}\right\},
$$

where the best fit parameters are [29]

$$
\begin{gathered}
g_{1}=0.201 \mathrm{GeV}^{2}, \quad g_{2}=0.184 \mathrm{GeV}^{2}, \quad g_{3}=-0.129, \\
Q_{0}=1.6 \mathrm{GeV}, \quad b_{\max }=1.5 \mathrm{GeV}^{-1}, \quad x_{0}=0.009, \quad x=0.09 .
\end{gathered}
$$

So far no experimental data is available to extract the nonperturbative fitting parameters of linearly polarized gluon TMD. Hence the same nonperturbative Sudakov factor is chosen for linearly polarized gluon TMD pdf which is considered for unpolarized distribution function. However, as discussed in [29], the $Q$ independent part of the nonperturbative Sudakov factor is expected to depend on spin, so one should in principle use a different $Q$-independent part for the linearly polarized gluon distribution; but the difference does not affect the result at large $Q$. The TMD distribution function $f\left(x, b_{\perp}, Q_{i}, \zeta\right)$ is formally written in terms of a product of convolution of coefficient function and standard collinear pdf [3]

$$
f\left(x, b_{\perp}, Q_{i}, \zeta\right)=\sum_{i=g, q} \int_{x}^{1} \frac{d \hat{x}}{\hat{x}} C_{i / g}\left(x / \hat{x}, b_{\perp}, \alpha_{s}, \mu, \zeta\right) f_{i / p}\left(\hat{x}, c / b_{*}\right)+\mathcal{O}\left(b_{\perp} \Lambda_{Q C D}\right)
$$


The coefficient function is calculated perturbatively which is different for each TMD pdf and independent of process. The collinear pdf produces the perturbative tail at small $b_{\perp}$ values which is evaluated at scale $c / b_{*}$ rather than $Q$ in contrast to the DGLAP evolution. We neglect quark contribution since it's effect is small compared to gluon. The unpolarized and linearly polarized TMD pdf at leading and first order in $\alpha_{s}$ are given by [29]

$$
\begin{gathered}
f_{1}^{g}\left(x, b_{\perp}, Q_{i}, \zeta\right)=f_{g / p}\left(x, c / b_{*}\right)+\mathcal{O}\left(\alpha_{s}\right) \\
h_{1}^{\perp g}\left(x, b_{\perp}, Q_{i}, \zeta\right)=\frac{\alpha_{s}\left(c / b_{*}\right) C_{A}}{\pi} \int_{x}^{1} \frac{d \hat{x}}{\hat{x}}\left(\frac{\hat{x}}{x}-1\right) f_{g / p}\left(\hat{x}, c / b_{*}\right)+\mathcal{O}\left(\alpha_{s}^{2}\right) .
\end{gathered}
$$

Now we can write the Eq.(34) as the following by using Sudakov factors and TMD pdfs

$$
\frac{d^{2} \sigma^{f f+h h}}{d y d q_{T}^{2}}=\frac{d^{2} \sigma^{f f}}{d y d q_{T}^{2}}+\frac{d^{2} \sigma^{h h}}{d y d q_{T}^{2}}
$$

where

$$
\begin{aligned}
\frac{d^{2} \sigma^{f f}}{d y d q_{T}^{2}}= & \frac{\rho}{36 s} \int_{4 m_{Q}^{2}}^{4 m_{Q \bar{q}}^{2}} d M^{2} \int_{0}^{\infty} b_{\perp} d b_{\perp} J_{0}\left(q_{T} b_{\perp}\right) f_{1}^{g}\left(x_{a}, c / b_{*}\right) f_{1}^{g}\left(x_{b}, c / b_{*}\right) \hat{\sigma}^{g g \rightarrow Q \bar{Q}}\left(M^{2}\right) \\
& \exp \left\{-2 \int_{c / b_{*}}^{Q} \frac{d \mu}{\mu}\left(A \log \left(\frac{Q^{2}}{\mu^{2}}\right)+B\right)\right\} \exp \left\{-\left[0.184 \log \frac{Q}{2 Q_{0}}+0.332\right] b_{\perp}^{2}\right\},
\end{aligned}
$$

and

$$
\begin{aligned}
\frac{d^{2} \sigma^{h h}}{d y d q_{T}^{2}}= & \frac{\rho C_{A}^{2}}{36 s \pi^{2}} \int_{4 m_{Q}^{2}}^{4 m_{Q \bar{q}}^{2}} d M^{2} \int_{0}^{\infty} b_{\perp} d b_{\perp} J_{0}\left(q_{T} b_{\perp}\right) \alpha_{s}^{2}\left(c / b_{*}\right) \hat{\sigma}^{g g \rightarrow Q \bar{Q}}\left(M^{2}\right) \\
& \int_{x_{a}}^{1} \frac{d x_{1}}{x_{1}}\left(\frac{x_{1}}{x_{a}}-1\right) f_{1}^{g}\left(x_{1}, c / b_{*}\right) \int_{x_{b}}^{1} \frac{d x_{2}}{x_{2}}\left(\frac{x_{2}}{x_{b}}-1\right) f_{1}^{g}\left(x_{2}, c / b_{*}\right) \\
& \exp \left\{-2 \int_{c / b_{*}}^{Q} \frac{d \mu}{\mu}\left(A \log \left(\frac{Q^{2}}{\mu^{2}}\right)+B\right)\right\} \exp \left\{-\left[0.184 \log \frac{Q}{2 Q_{0}}+0.332\right] b_{\perp}^{2}\right\}
\end{aligned}
$$

\section{NUMERICAL RESULTS}

In this section we present our numerical results. As for CEM, the squared invariant mass of the quark pair is integrated from $4 m_{Q}^{2}$ to $4 m_{Q \bar{q}}^{2}$. For $J / \psi$ production, we have taken charm quark mass $\left(m_{c}=1.275 \mathrm{GeV}\right)$ for $m_{Q}$ and lightest D meson mass $\left(m_{D}=1.863 \mathrm{GeV}\right)$ for $m_{Q \bar{q}}$. Moreover, bottom quark mass $\left(m_{b}=4.18 \mathrm{GeV}\right)$ for $m_{Q}$ and lightest B meson mass $\left(m_{B}=5.279\right.$ 
$\mathrm{GeV}$ ) for $m_{Q \bar{q}}$ have been considered for $\Upsilon$ production. MSTW2008 [51] has been used for pdfs to obtain the differential cross section.

We have calculated the LO transverse momentum $\left(q_{T}\right)$ and rapidity $(y)$ distributions of $J / \psi$ and $\Upsilon$, we also present the cross section differential in $q_{T}$ using TMD evolution formalisms at center-of mass energies of LHCb $(\sqrt{s}=7 \mathrm{TeV})$, RHIC $(\sqrt{s}=500 \mathrm{GeV})$ and AFTER $(\sqrt{s}=115$ $\mathrm{GeV})$ experiments. The rapidity of quarkonium is integrated in the range of $y \in[2.0,4.5]$, $y \in[-3.0,3.0]$ and $y \in[-0.5,0.5]$ for LHCb, RHIC and AFTER respectively, to obtain the differential cross section as a function of $q_{T}$. The rapidity distribution of quarkonium has been calculated by integrating $q_{T}$ from 0 to $0.5 \mathrm{GeV}$ for all energies. The conventions in the figures are the following. In all the figures, "ff" denotes contributions of unpolarized TMDs only in the cross section and "ff $+\mathrm{hh}$ " means both unpolarized pdfs and linearly polarized gluon distributions are taken into account.

The transverse momentum and rapidity distributions have been estimated in Model-I from Eq.(25) \& (26) and Model-II from Eq.(27) \& (28) by employing a Gaussian model. In Figs. 2 and 3 we have divided the result by the total cross section for the kinematics of each experiment, as a result, we got overlapping curves independent of the center-of-mass energy of the experiment and the mass of the quarkonium. For different values of Gaussian width $\left\langle k_{\perp}^{2}\right\rangle$ and parameter $r$ the $q_{T}$ distribution of $J / \psi$ and $\Upsilon$ has been shown in Fig. 2 and 3. Fig. 2 is for $r=2 / 3$ and Fig. 3 is for $r=1 / 3$. The invariant mass square of the heavy quark pair $\left(M^{2}\right)$ which has very narrow range from $4 m_{Q}^{2}$ to $4 m_{Q \bar{q}}^{2}$ in this model is used as a scale to evolve the pdfs for all experiments using DGLAP evolution equation in these plots. We have noticed that linearly polarized gluons does not contribute to integrated $q_{T}$ cross section of charmonium and bottomonium in DGLAP approach. The transverse momentum distribution of $J / \psi$ and $\Upsilon$ in Model-I is shown in Fig. 2 and 3 are in agreement with results [25] for $\chi_{c, b 0}$ quarkonium production obtained by NRQCD framework. It is seen in the Fig. 2 and 3 that the inclusion of linearly polarized gluon contribution to the unpolarized gluon cross section have greatly modulated the transverse momentum distribution of charmonium and bottomonium mostly at low $q_{T}, q_{T}<0.5 \mathrm{GeV}$. So measuring the cross section of charmonium production at low transverse momentum can help to disentangle the linearly polarized gluon contribution. The transverse momentum dependent cross section of quarkonium is higher in Model-II compared 
to Model-I. In Fig. 3, we have also shown the $q \bar{q}$ contribution in quarkonium production. This is extremely small, compared to the gluon channel in the kinematics of the experiments considered. Therefore we have not considered contribution of this channel in other plots.

Rapidity distribution of charmonium and bottomonium has been obtained in Model-I and II using DGLAP evolution which is shown in figures 4-6. Fig. 4 is for the kinematics of LHCb, Fig. 5 is for RHIC and Fig. 6 is for the kinematics of AFTER. We have chosen different rapidity range for different experiments, that are given above. In order to show the effect of linearly polarized gluons, we have chosen a small $q_{T}$ bin, namely $0<q_{T}<0.5 \mathrm{GeV}$ in all these plots. The cross section decreases with increasing rapidity. Rapidity distribution is enhanced by considering the linearly polarized gluons apart from unpolarized gluons in the cross section. Moreover, the enhancement is more in Model-I compared to Model-II. Furthermore, we have noticed that the $y$ distribution is independent of parameter $r$ and Gaussian width $\left\langle k_{\perp}^{2}\right\rangle$.

Within TMD evolution formalism using Eq.(42), the transverse momentum dependent cross section of quarkonium is shown in figures 7-9. Fig. 7 is for the kinematics of LHCb, Fig. 8 is for RHIC and Fig. 9 is for AFTER. In theses plots, we have integrated over the rapidity in the ranges given above for different experiments. The effect of $h_{1}^{\perp g}$ in the $q_{T}$ distribution in the TMD evolution approach is not as dominant as in the DGLAP evolution approach, particularly for $\Upsilon$, although it is sizable at low $q_{T}$. The mass of the $\Upsilon$ is more than that of $J / \psi$, and this effect is suppressed by the mass. Only one loop in $\alpha_{s}$ has been taken to integrate the Sudakov factor in Eq.(36). The transverse momentum distribution of quarkonium is reduced in TMD evolution formalism compared to DGLAP evolution formalism. Fig. 10 represents the decline of $q_{T}$ distribution in TMD approach in contrast to DGLAP. Here we have chosen the kinematics of LHCb experiment. The rapidity is integrated over the region $y \in[2.0,4.5]$ TMD pdfs are evolved in TMD evolution from initial scale $\left(c / b_{*}\right)$ to final scale $(Q)$, where $Q$ has been set equal to quarkonium mass i.e., $Q=M$ which is the relevant scale for production of charmonium and bottomonium. 

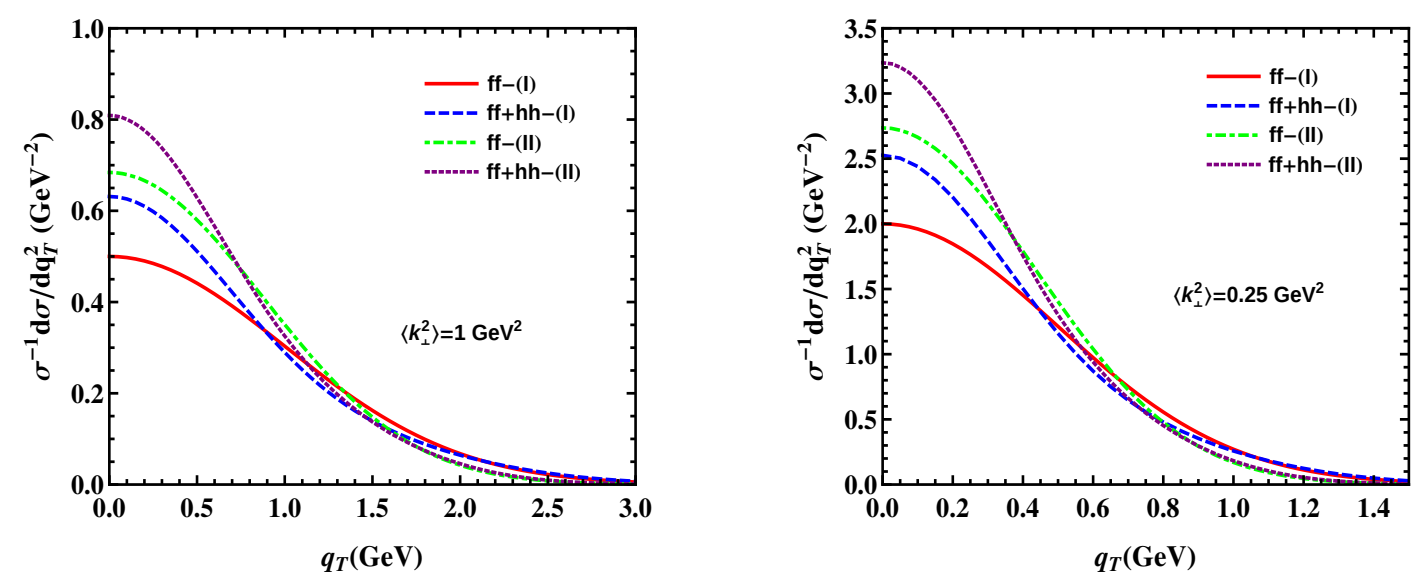

FIG. 2: (color online) Differential cross section (normalized) of $J / \psi$ and $\Upsilon$ production in pp $\rightarrow Q \bar{Q}+X$ at $\operatorname{LHCb}(\sqrt{s}=7 \mathrm{TeV})$, RHIC $(\sqrt{s}=500 \mathrm{GeV})$ and AFTER $(\sqrt{s}=115 \mathrm{GeV})$ energies using DGLAP evolution approach For $r=\frac{2}{3}$. The solid (ff-(I)) and dot dashed (ff-(II)) lines are obtained by considering unpolarized gluons and quarks in Model-I and Model-II respectively. The dashed (ff+hh(I)) and tiny dashed (ff+hh-(II)) lines are obtained by taking into account unpolarized gluons and quarks plus linearly polarized gluons in Model-I and Model-II respectively. See the text for ranges of rapidity integration.
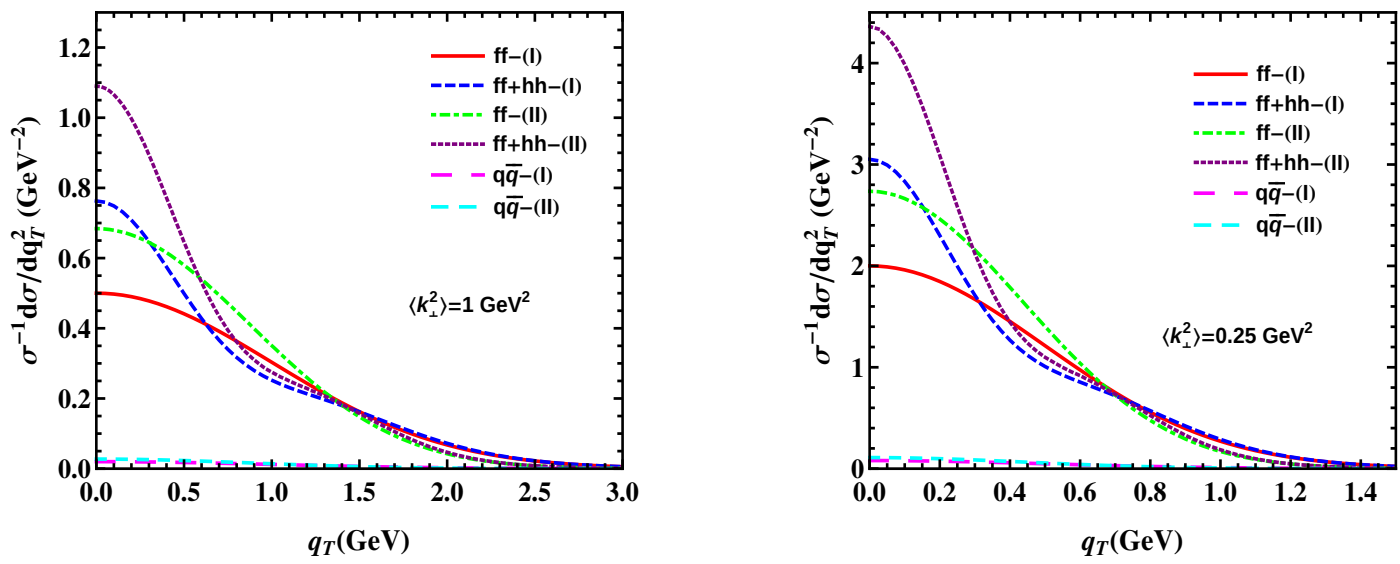

FIG. 3: (color online) Same as in Fig. 2 but for $r=\frac{1}{3}$. 


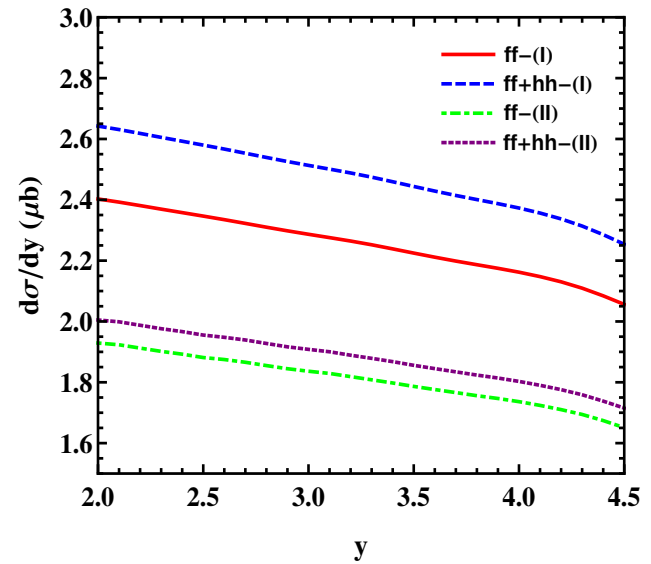

(a)

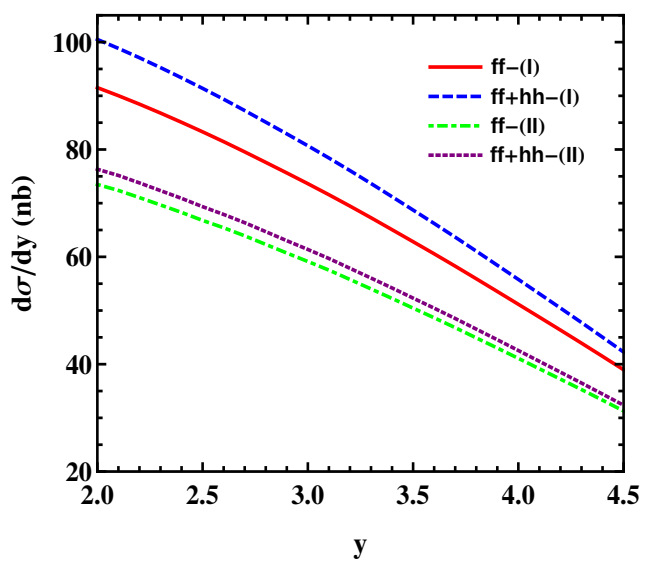

(b)

FIG. 4: (color online) Rapidity (y) distribution of (a) $J / \psi$ (left panel) and (b) $\Upsilon$ (right panel) in $\mathrm{pp} \rightarrow Q \bar{Q}+X$ at $\mathrm{LHCb}(\sqrt{s}=7 \mathrm{TeV})$ energy and $q_{T}$ integration range is from 0 to $0.5 \mathrm{GeV}$ using DGLAP evolution approach. The convention in the figure for line styles is same as Fig. 2.

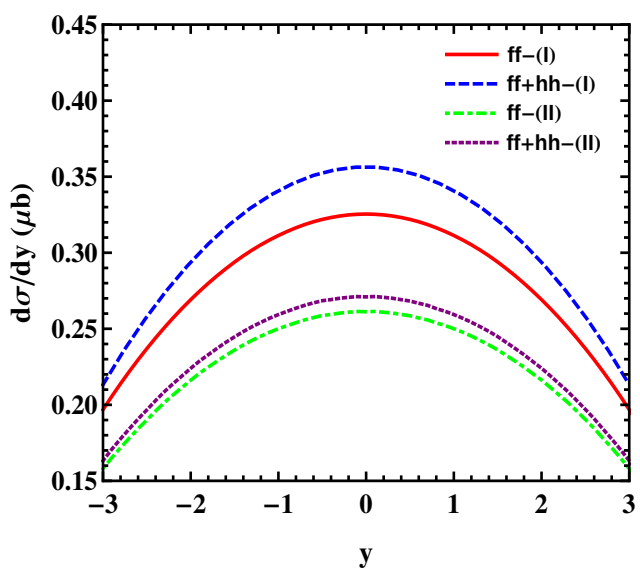

(a)

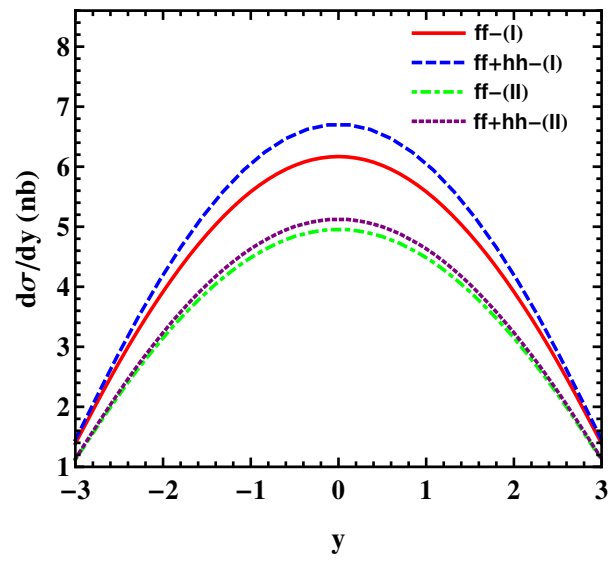

(b)

FIG. 5: (color online) Rapidity (y) distribution of (a) $J / \psi$ (left panel) and (b) $\Upsilon$ (right panel) in $\mathrm{pp} \rightarrow Q \bar{Q}+X$ at RHIC $(\sqrt{s}=500 \mathrm{GeV})$ energy and $q_{T}$ integration range is from 0 to $0.5 \mathrm{GeV}$ using DGLAP evolution approach. The convention in the figure for line styles is same as Fig. 2. 


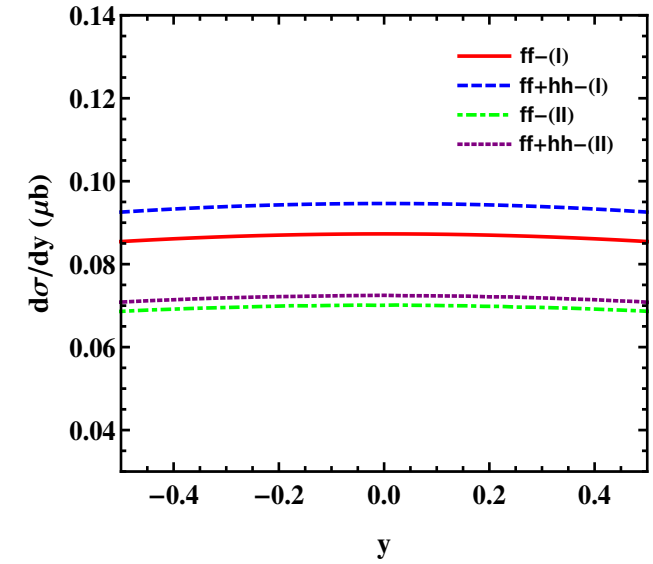

(a)

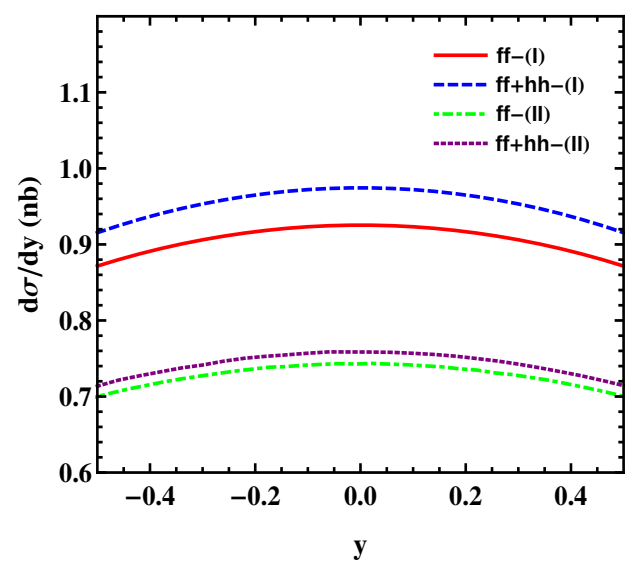

(b)

FIG. 6: (color online). Rapidity (y) distribution of (a) $J / \psi$ (left panel) and (b) $\Upsilon$ (right panel) in $\mathrm{pp} \rightarrow Q \bar{Q}+X$ at AFTER $(\sqrt{s}=115 \mathrm{GeV})$ energy and $q_{T}$ integration range is from 0 to $0.5 \mathrm{GeV}$ using DGLAP evolution approach. The convention in the figure for line styles is same as Fig. 2.

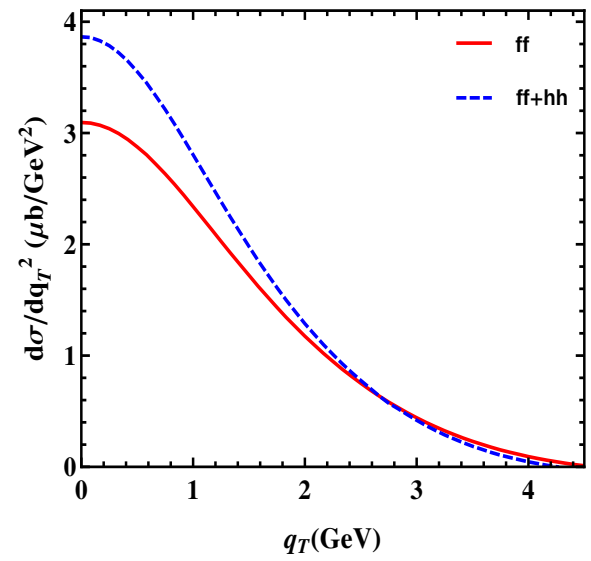

(a)

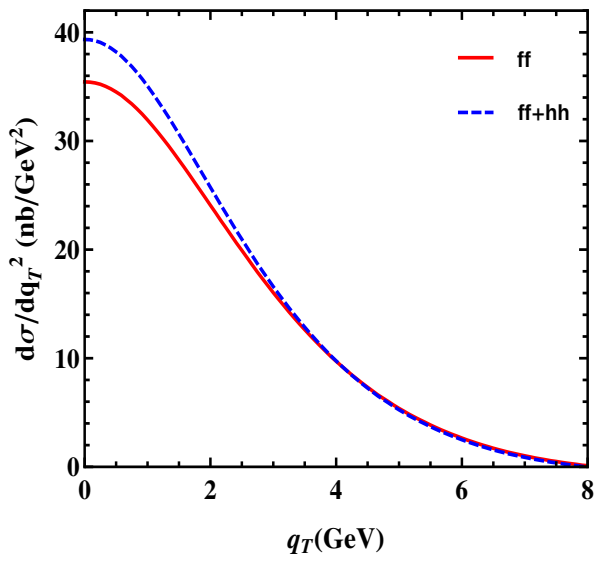

(b)

FIG. 7: (color online). Differential cross section of (a) $J / \psi$ (left panel) and (b) $\Upsilon$ (right panel) as function of $q_{T}$ in $\mathrm{pp} \rightarrow Q \bar{Q}+X$ at $\mathrm{LHCb}(\sqrt{s}=7 \mathrm{TeV})$ energy using TMD evolution approach. The solid (ff) and dashed ( $\mathrm{ff}+\mathrm{hh}$ ) lines are obtained by considering unpolarized gluons only and unpolarized plus linearly polarized gluons respectively. 


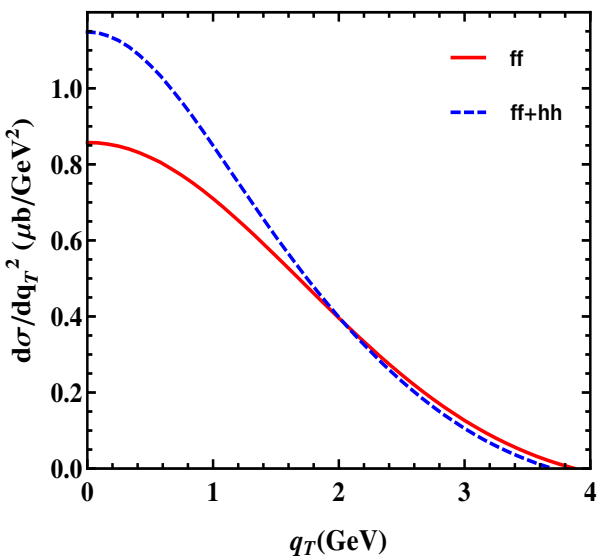

(a)

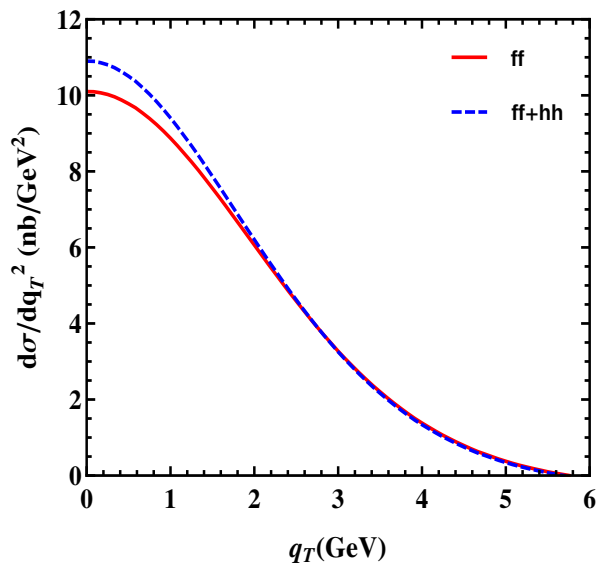

(b)

FIG. 8: (color online). Differential cross section of (a) $J / \psi$ (left panel) and (b) $\Upsilon$ (right panel) as function of $q_{T}$ in $\mathrm{pp} \rightarrow Q \bar{Q}+X$ at RHIC $(\sqrt{s}=500 \mathrm{GeV})$ energy using TMD evolution approach. The convention in the figure for line styles is same as Fig. 7.

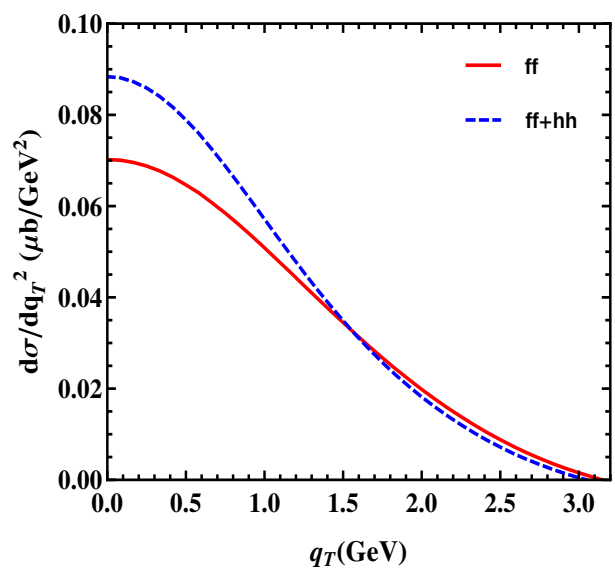

(a)

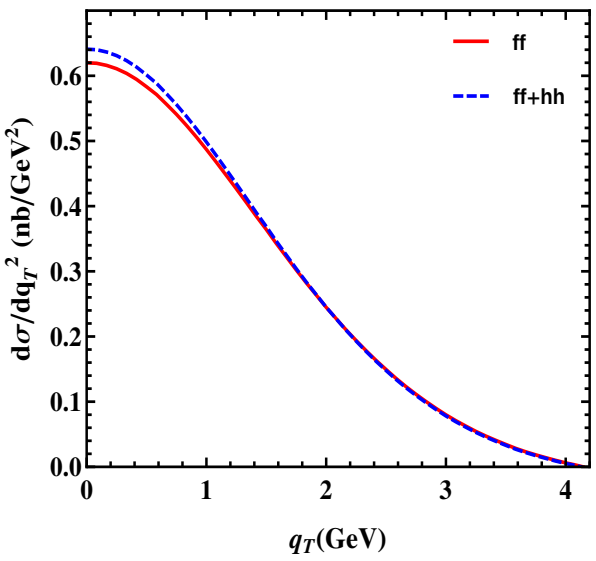

(b)

FIG. 9: (color online). Differential cross section of (a) $J / \psi$ (left panel) and (b) $\Upsilon$ (right panel) as function of $q_{T}$ in pp $\rightarrow Q \bar{Q}+X$ at AFTER $(\sqrt{s}=115 \mathrm{GeV})$ energy using TMD evolution approach. The convention in the figure for line styles is same as Fig. 7. 


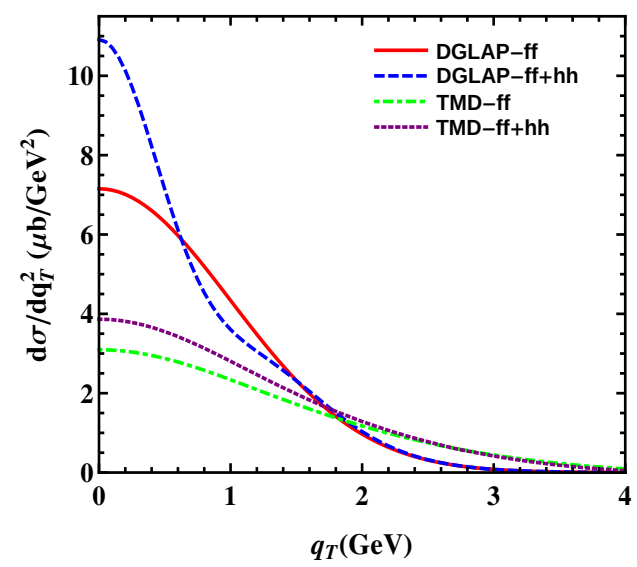

(a)

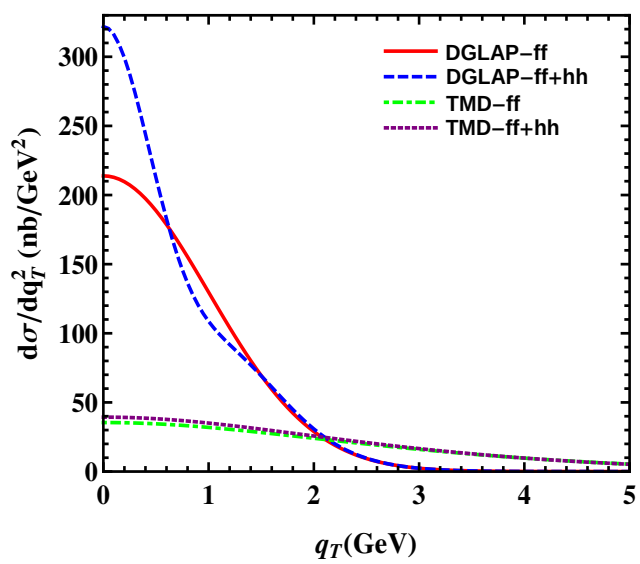

(b)

FIG. 10: (color online). Differential cross section of (a) $J / \psi$ (left panel) and (b) $\Upsilon$ (right panel) in $\mathrm{pp} \rightarrow Q \bar{Q}+X$ at LHCb $(\sqrt{s}=7 \mathrm{TeV})$. The solid (DGLAP-ff) and dot dashed (TMD-ff) lines are obtained by considering unpolarized gluons and quarks in DGLAP and only unpolarized gluons in TMD evolution respectively. The dashed (DGLAP-ff+hh) and tiny dashed (TMD-ff+hh) lines are obtained by taking into account unpolarized gluons and quarks plus linearly polarized gluons in DGLAP and unpolarized plus linearly polarized gluons in TMD evolution respectively. We have chosen $r=\frac{1}{3}$ and $\left\langle k_{\perp}^{2}\right\rangle=1 \mathrm{GeV}^{2}$ are taken in Model-I for DGLAP evolution.

\section{CONCLUSION}

Summarizing, we studied transverse momentum and rapidity distributions of $J / \psi$ and $\Upsilon$ in unpolarized proton-proton collision within the formalism of transverse momentum dependent factorization. Since a long time, a lot of efforts have been put forward to understand the hadronization of heavy quarks into mesons, both experimentally and theoretically. However, none of the models (CSM, NRQCD and CEM) could describe the transverse momentum dependent cross section of $J / \psi$ completely by fitting with experimental data [52]. Therefore, it would be interesting to include the linearly polarized gluon contribution in the cross section to fit the experimental data to the extent of reasonable accuracy. On the other hand, by combining data from different experiments in different kinematical regions one can quantify the magnitude of $h_{1}^{\perp g}$. Most experiments measure spin and azimuthal asymmetries to probe the transverse momentum dependent parton distributions and get information on the spin and angular mo- 
mentum structure of the hadrons. It is also important to get a quantitative understanding of the unpolarized gluon TMD $f_{1}^{g}\left(x, k_{\perp}\right)$. This lies at the denominator of the spin asymmetries and its contribution is important in small $x$ region that is expected to play an important role in collider experiments for example, in the future eRHIC. We employed color evaporation model (CEM), for its simplicity, to calculate the differential cross section of quarkonium production and to illustrate the effect of linearly polarized gluons distribution. We studied the effect of TMD evolution at LHCb, RHIC and AFTER energies. We found that contribution from the $q \bar{q}$ channel is very small compared to the gluon channel. We observed that the inclusion of linearly polarized gluons significantly modulated the transverse momentum distribution of $J / \psi$ and $\Upsilon$ at low $q_{T}$. We further noticed that rapidity distribution has been enhanced by taking the presence of linearly polarized gluons into account inside the unpolarized proton. By this we conclude that charmonium and bottomonium production via gluon fusion process is a very useful tool to probe the unpolarized gluon TMD and linearly polarized gluons distribution.

\section{ACKNOWLEDGEMENTS}

S.Rajesh acknowledges Sreeraj Nair for his help in Fortran code. Vikash Kumar Ojha is thanked for his help rendered during initial stage of this project.

\section{References}

[1] J. Collins, Foundations of Perturbative QCD, Cambridge University Press, 2011.

[2] Stefano Melis, EPJ Web Conf. 85 (2015) 01001.

[3] S.M. Aybat, T.C. Rogers, Phys. Rev. D 83 (2011) 114042.

[4] D. Boer and P. J. Mulders, Phys. Rev. D 57, 5780 (1998).

[5] P. J. Mulders and J. Rodrigues, Phys. Rev. D 63, 094021 (2001).

[6] S. Falciano et al. (NA10), Z. Phys. C 31, 513 (1986).

[7] M. Guanziroli et al. (NA10), Z. Phys. C 37, 545 (1988).

[8] M. Arneodo et al. (EMC), Z. Phys. C 34, 277 (1987). 
[9] J. Breitweg et al. (ZEUS), Phys. Lett. B 481, 199 (2000).

[10] D. Boer, Phys. Rev. D 60, 014012 (1999).

[11] V. Barone, S. Melis, and A. Prokudin, Phys. Rev. D 81,114026 (2010).

[12] D. W. Sivers, Phys. Rev. D 41, 83 (1990).

[13] D. W. Sivers, Phys. Rev. D 43, 261 (1991).

[14] R. N. Cahn, Phys. Lett. 78B, 269 (1978).

[15] R. N. Cahn, Phys. Rev. D40, 3107 (1989).

[16] V. Barone, S. Melis, and A. Prokudin, Phys. Rev. D 82,114025 (2010).

[17] V. Barone, M. Boglione, J. O. Gonzalez Hernandez, and S. Melis, Phys. Rev. D 91, 074019 (2015).

[18] D. Boer, S.J. Brodsky, P.J. Mulders and C. Pisano, Phys. Rev. Lett. 106, 132001 (2011).

[19] Jian-Wei Qiu, Marc Schlegel and Werner Vogelsang, Phys. Rev. Lett. 107, 062001 (2011).

[20] Cristian Pisano, Daniel Boer, Stanley J. Brodsky, Maarten G.A. Buffing and Piet J. Mulders, JHEP, 10 (2013) 024.

[21] Daniel Boer, Piet J. Mulders, and Cristian Pisano, Phys. Rev. D 80, 094017 (2009).

[22] P. Sun, B.-W. Xiao and F. Yuan,Phys. Rev. D 84, 094005 (2011).

[23] D. Boer, W.J. den Dunnen, C. Pisano, M. Schlegel and W. Vogelsang, Phys. Rev. Lett. 108 032002 (2012).

[24] D. Boer, W.J. den Dunnen, C. Pisano and M. Schlegel, Phys. Rev. Lett. 111032002 (2013).

[25] Daniel Boer and Cristian Pisano, Phys. Rev. D 86, 094007 (20012).

[26] A. Ito, R. Fisk, H. Jostlein, D. Kaplan, S. Herb et al., Phys.Rev. D23,604 (1981).

[27] T. Aaltonen et al. (CDF Collaboration), Phys.Rev. D 86, 052010 (2012), 1207.7138.

[28] M. G. Echevarria, A. Idilbi, Z.-B. Kang, and I. Vitev, Phys.Rev. D 89, 074013 (2014).

[29] D. Boer and W. J. den Dunnen, Nucl. Phys. B 886 (2014) 421.

[30] E. L. Berger and D. L. Jones, Phys. Rev. D 23, 1521 (1981); R. Baier and R. Ruckl, Phys. Lett. B 102, 364 (1981); R. Baier and R. Ruckl, Nucl. Phys. B 201, 1 (1982).

[31] N. Brambilla et al., Eur. Phys. J. C 71, 1534 (2011).

[32] G. T. Bodwin, E. Braaten, and J. Lee, Phys. Rev. D 72,014004 (2005).

[33] G. T. Bodwin, E. Braaten, G. P. Lepage, Phys. Rev. D 46, 1914 (1992).

[34] F. Halzen, Phys. Lett. B 69, 105 (1977); F. Halzen and S. Matsuda, Phys. Rev. D 17, 1344 (1978). 
[35] H. Fritsch, Phys. Lett. B 67, 217 (1977).

[36] G. A. Schler and R. Vogt, Phys. Lett. B 387, 181 (1996).

[37] J. F. Amundson, O. J. P. Eboli, E. M. Gregores and F. Halzen, Phys. Lett. B 372, 127 (1996).

[38] Chrisopher P. Smith, "Heavy Quarkonium Hadroproduction in the Color Evaporation Model", M.Sc thesis, University of Wisconsin, 2000.

[39] O. J. P. Eboli, E. M. Gregores and F. Halzen, Phys. Rev. D 67, 054002 (2003).

[40] M. B. Gay Ducati and C. Brenner Mariotto, Phys. Lett. B 464, 286 (1999).

[41] R. Godbole, A. Misra, A. Mukherjee, V. Rawoot, Phys. Rev. D 85, 094013 (2012).

[42] R. Godbole, A. Misra, A. Mukherjee, V. Rawoot, Phys. Rev. D 88, 014029 (2013).

[43] D. Boer, P. J. Mulders, and C. Pisano, Phys. Lett. B 660, 360 (2008).

[44] M. Anselmino, U. DAlesio, and F. Murgia, Phys. Rev. D 67, 074010 (2003).

[45] M. Gluck and E. Reya, Phys. Lett. 79 B, 453 (1978).

[46] M. Anselmino, M. Boglione, U. DAlesio, A. Kotzinian, F. Murgia, and A. Prokudin, Phys. Rev. D 72, 094007 (2005).

[47] M . Anselmino, M. Boglione, U. DAlesio, A. Kotzinian,S. Melis, F. Murgia, A. Prokudin, and C. Turk, Eur. Phys. J. A 39, 89 (2009).

[48] S.M. Aybat, A. Prokudin, T.C. Rogers, Phys. Rev. Lett. 108 (2012) 242003.

[49] A. Idilbi, X.-d. Ji, and F. Yuan, Nucl. Phys. B753, 42 (2006).

[50] P. Sun, B.-W. Xiao, F. Yuan, Phys. Rev. D 84 (2011) 094005.

[51] A.D. Martin, W.J.Stirling, R.S. Thorne, G.Watt, Eur. Phys. J. C (2009) 63: 189285.

[52] R. Aaij et al. (LHCb collaboration), Eur. Phys. J . C 71 (2011) 1645. 